\title{
Assessing Patient Experience and Orientation in the Emergency Department with Virtual Windows
}

\author{
Yonatan G Keschner \\ Department of Emergency Medicine \\ Brigham and Women's Hospital \\ Harvard Medical School \\ ykeschner@bwh.harvard.edu \\ Christopher W Baugh \\ Department of Emergency Medicine \\ Brigham and Women's Hospital \\ Harvard Medical School \\ cbaugh@bwh.harvard.edu
}

\author{
Adam B Landman \\ Brigham Digital Innovation Hub \\ Department of Emergency Medicine \\ Brigham and Women's Hospital \\ Harvard Medical School \\ alandman@bwh.harvard.edu
}

\author{
Mohammad Adrian Hasdianda \\ Department of Emergency Medicine \\ Brigham and Women's Hospital \\ Harvard Medical School \\ mhasdianda@bwh.harvard.edu \\ Paul C Chen \\ Department of Emergency Medicine \\ Brigham and Women's Hospital \\ Harvard Medical School \\ pchen@bwh.harvard.edu
}

Peter R Chai

Department of Emergency Medicine

Brigham and Women's Hospital

The Fenway Institute

Harvard Medical School

pchai@bwh.harvard.edu

\author{
Steven Miyawaki \\ Department of Emergency Medicine \\ Brigham and Women's Hospital \\ smiyawaki@bwh.harvard.edu
}

\author{
Haipeng Mark Zhang \\ Brigham Digital Innovation Hub \\ Brigham and Women's Hospital \\ Dana-Farber Cancer Institute \\ Harvard Medical School \\ hzhang37@bwh.harvard.edu
}

\begin{abstract}
Patients have benefitted from increasingly sophisticated diagnostic and therapeutic innovations over the years. However, the design of the physical hospital environment has garnered less attention. This may negatively impact a patient's experience and health. In areas of the hospital, such as the emergency department (ED), patients may spend hours, or even days, in a windowless environment. Studies have highlighted the importance of natural light and imagery, as they are essential in providing important stimuli to regulate circadian rhythm and orientation, and to mitigate the onset of certain medical conditions. In hospital locations where standard windows may be infeasible, the use of a virtual window may simulate the benefits of an actual window. In this pilot study, we assessed patient experience and orientation with virtual windows in the ED. We demonstrated that virtual windows are an acceptable technology that may improve patient experience and orientation.
\end{abstract}

\section{Introduction}

Patients spend a significant amount of time in the hospital without access to the outdoors and natural light. Instead, hospitalized individuals are mostly exposed to indoor lighting which remains at a constant intensity despite circadian changes in lighting outdoors. In settings where windows are physically unavailable, such as rooms without externally facing walls commonly encountered in the emergency department (ED), there can be significant morbidity associated with lack of exposure to the outdoors $[1,2]$. Most importantly, natural light and views of the outdoors provide important stimuli that calibrate circadian rhythm and orientation [3]. The lack of exposure to natural light results in increased risk of delirium, disorientation, and decreased satisfaction with the hospital experience [4, 5]. For example, within the intensive care unit (ICU), the lack of exposure to natural light and the outdoors mars normal circadian rhythm and sleep resulting in ICU delirium and insomnia. While many hospitals institute lighting changes to mimic sleep wake cycles, there is also an additional benefit to having outdoor exposure. One ICU investigation found there were twice as many episodes of delirium in patients admitted to windowless rooms compared to rooms with windows [6]. Another prospective ICU study comparing patients admitted to a room with or without exposure to natural light from windows found that patients exposed to a window had reduced risk of episodes of agitation and hallucinations [7].

The ED is a unique location in the hospital where the lack of outdoor stimuli is ubiquitous. Due to its role as a department which manages new patients 24 hours a day, the ED has near continuous lighting with 
minimal changes in light intensity or duration. These features, while necessary to provide ongoing critical care, may also be deleterious to patients, and exacerbate delirium, especially among those who are elderly and individuals with existing disorientation due to medical and psychiatric conditions. Patients who suffer from acute disorientation may suffer from key morbidity and even mortality which can lead to hospitalizations and worsening disease. For individuals who remain in the ED for long periods of time due to inpatient hospital crowding or psychiatric comorbidities, the lack of natural cycles of lighting may contribute to increased paranoia and agitation [8].

Virtual windows have been developed as a novel approach to substitute the benefits of an actual window by providing natural light and outdoor views in an otherwise windowless environment [9]. These systems are comprised of a light emitting diode (LED) screen linked to a small controller and an incandescent light box that emits natural spectrum of light that mimics outdoor lighting. The controller can be linked wirelessly to an outdoor camera to provide live feeds of the outdoors virtually into the window, or it can play a pre-recorded loop of a video clip. The light box is linked to the video feed thereby allowing an additional visual stimulus of either gradations of light intensity as the day progresses, or a simple on and off function to turn off the light at night. Unlike a LED screen that plays a video of the outdoors, the combination of a LED screen with a lighting box provides both a visual and physical stimulus to the user. While the use of physical window spaces have been demonstrated to improve orientation and address key outcomes in inpatients, no studies have considered the use of a virtual window to provide outdoor views and orientation in hospital settings where it is impossible or infeasible to place physical windows. In this study, we deployed a virtual window in the ED and sought to understand the acceptability of these systems among ED patients and perceptions surrounding their benefit to increase patient satisfaction.

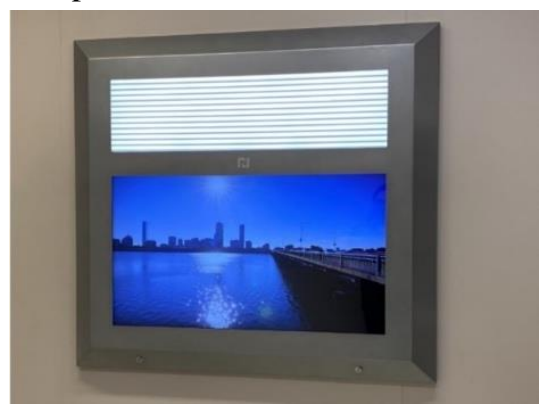

Figure 1. Virtual Window. A virtual window with a metal, tamper proof frame containing a natural light box and high-definition monitor (dimensions of virtual window frame $114 \mathrm{~cm} \times 127 \mathrm{~cm})$.

\section{Methods}

We conducted a prospective cohort study based at an academic, urban, tertiary-care level care center with more than 65,000 annual adult ED visits. We selected three rooms in the ED that are designed for both medical and psychiatric patients. We installed three virtual windows (Novolux, Newton MA) on walls and used a ligature-resistant encasement to prevent damage to electronic components or harm from the device to ED patients (Figure 1). Next, we loaded the virtual window with a pre-recorded video stream that displayed a bridge over a well-recognized river in the city from the hours of 9 am to $5 \mathrm{pm}$. We elected to utilize prerecorded video to avoid technical barriers and reduce information security risk that would have existed if we had integrated live video feeds for the virtual windows into our hospital network. We synchronized the video with the time of day to permit the virtual window to play a continuous video loop from $9 \mathrm{am}$ to $5 \mathrm{pm}$. During the time when the video was playing on the virtual window, we additionally turned on the lightbox to provide ambient light. At 5pm each day, the video from the window and the lightbox automatically turned off. This study was approved by the Mass General Brigham Institutional Review Board.

We enrolled a convenience sample of patients over 18 years of age presenting for care in the ED, during the daytime hours of $7 \mathrm{am}$ to $1 \mathrm{pm}$. Potentially eligible patients who were placed by ED triage staff into a room with a virtual window had eligibility criteria reviewed by a trained study team member. Inclusion criteria included age over 18 years old, English speaking, and medically stable and able to participate in the study procedures. Exclusion criteria included unable to provide informed consent, dementia or other mental impairment, suspected/active COVID-19 infection, medically unstable, or previously consented for the study. Next, we approached participants, explained the study, and obtained verbal consent to participate in the study. We collected data on participants demographics and diagnosis from the electronic health record (EHR) system. Participants received a quantitative assessment at enrollment consisting of the Media Technology and Usage Scale (MTUAS) with positive and negative attitudes associated with technology [5point Likert scale, Strongly disagree (1) to Strongly agree (5)] [10]. Participants were asked at the baseline and exit surveys questions related to their orientation without the aid of a clock. Participants completed the exit survey when their disposition order was placed in the EHR or notified by the primary clinical team. At the exit survey, we asked additional questions 
surrounding the participant experience interacting with the virtual window during their stay, its obtrusiveness, impact on orientation and preference to be placed in a room with a virtual window. We utilized the validated, Perceived Stress Scale (PSS) to measure the participants' perception of stress at baseline in the last 30 days (10 questions on a 5-point Likert-like scale, Never to Very Often) [11].

\subsection{Data analysis}

We calculated basic descriptive statistics of study participants, including age, gender, diagnosis type. For MTUAS, we summed each question and calculated mean scores and report standard deviations. For discrete variable questions surrounding participant experience, we reported the frequency of each response.

\section{Results}

Over the study period, we screened 103 individuals (Figure 2). Of these, 40 individuals met eligibility criteria and were approached to enroll in the study; 19 of these individuals consented to participate. Fourteen participants completed all study measures while four participants only completed the baseline survey. One participant decided to discontinue participation while completing the baseline survey.

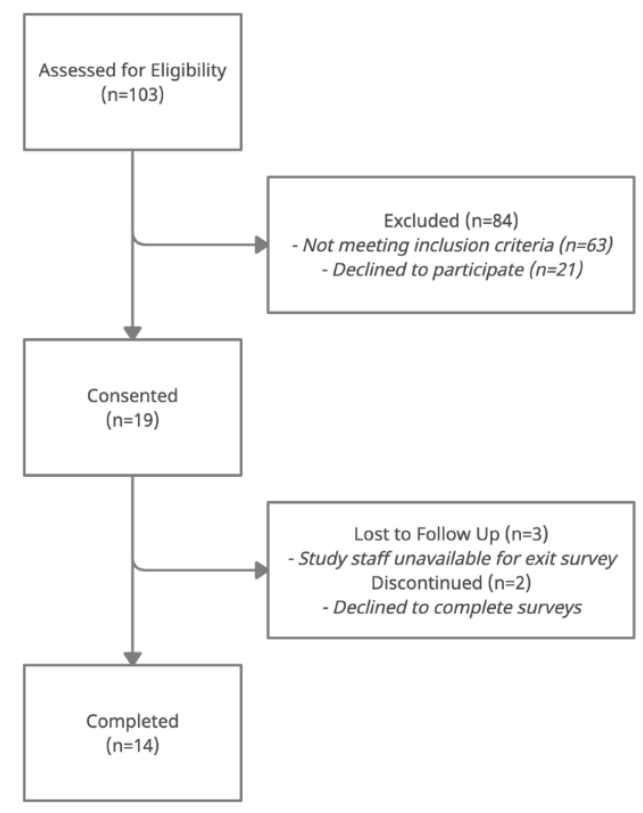

Figure 2. Study flow diagram

Mean age of participants was 49 years and 50\% (7) participants were female (Table 1). 10 participants had medical diagnoses and 4 had psychiatric diagnoses. On average, at baseline the participants had a moderate amount of perceived stress in the last month as measured by the Perceived Stress Scale for both medical and psychiatric patients. Participants reported overall positive attitudes towards technology (Mean score $3.8 \pm 0.9$ ).

Table 1. Participants' characteristics

\begin{tabular}{|c|c|}
\hline Age, years (mean, SD) & $48.9(16.4)$ \\
\hline Female $(\mathrm{n}, \%)$ & $7(50)$ \\
\hline \multicolumn{2}{|l|}{ Diagnosis type $(\mathrm{n}, \%)$} \\
\hline Medical & $10(71.4)$ \\
\hline Psychiatric & $4(28.6)$ \\
\hline Exposure to VW*, hours (mean, SD) & $5.0(2.4)$ \\
\hline \multicolumn{2}{|l|}{ Baseline PSS $^{\#}$ score (mean, SD) } \\
\hline Medical & $19.0(5.6)$ \\
\hline Psychiatric & $24.0(8.3)$ \\
\hline \multicolumn{2}{|l|}{ Device ownership $(\mathrm{n}, \%)$} \\
\hline Smartphone & $13(92.9)$ \\
\hline Laptop/computer & $9(64.3)$ \\
\hline \multicolumn{2}{|l|}{ MTUAS $^{\dagger}$ subscale score (mean, SD) } \\
\hline Positive attitudes to technology & $3.8(0.9)$ \\
\hline Anxiety of being without technology & $3.4(0.8)$ \\
\hline \multirow{3}{*}{\multicolumn{2}{|c|}{$\begin{array}{l}\text { *Virtual window } \\
\text { \#Perceived Stress Scale (0-13: low stress, 14-26 } \\
\text { moderate stress, 27-40: high stress) } \\
{ }^{\dagger} \text { Media and Technology Usage and Attitudes Scale } \\
\text { (5: strongly agree, 4: agree, 3: neither agree nor } \\
\text { disagree, 2: disagree, 1: strongly disagree) }\end{array}$}} \\
\hline & \\
\hline & \\
\hline \multicolumn{2}{|l|}{ 3.1. Quantitative assessment results } \\
\hline \multicolumn{2}{|c|}{$\begin{array}{l}\text { The baseline survey demonstrated that all } \\
\text { participants }(\mathrm{N}=14) \text { knew the time of day and if it was } \\
\text { dark or light outside without looking at the time (Table } \\
\text { 2). At the final study assessment, we found that nearly } \\
\text { all patients were oriented to time of day }(\mathrm{N}=13,93 \%) \\
\text { and if it was dark or light outside }(\mathrm{N}=13,93 \%) \text {. Only } \\
\text { two participants were unable to identify the time of } \\
\text { day-- one participant answered it was the afternoon, } \\
\text { when it was in fact, it was the evening and another } \\
\text { responded that it was dark outside when it was still } \\
\text { daylight. }\end{array}$} \\
\hline
\end{tabular}

Table 2. Orientation

\begin{tabular}{lcc}
\hline & \multicolumn{2}{c}{ Correct response (n, \%) } \\
& Baseline & Exit \\
\hline What time is & $14 / 14(100)$ & $13 / 14$ \\
it? & & $(92.9)$ \\
\hline Dark or light & $14 / 14(100)$ & $13 / 14$ \\
outside? & & $(92.9)$ \\
\hline
\end{tabular}


The final study assessment demonstrated that participants were mostly satisfied with the virtual windows (Table 3). Most patients $(\mathrm{N}=11,79 \%)$ responded that they like the virtual window from "quite a bit to extremely" and the remainder $(\mathrm{N}=3$, $21 \%$ ) responded "moderately". None of the patients responded that they did not like the virtual windows. The virtual windows were found to not interfere with patient's ability to rest, and only 2 patients found it to be distracting. Nearly all $(\mathrm{N}=11,79 \%)$ of patients responded that they would prefer to have a room with a virtual window versus a standard room without a virtual window. Of the 14 patients, 5 patients responded to the open response feedback question. Four of the patients made positive statements, including "it was calming" and "the window helped me stay grounded." The one negative comment stated, "distracting to people suffering from visual hallucinations."

Table 3. Virtual window (VW) experience

\begin{tabular}{|c|c|c|c|}
\hline & \multicolumn{3}{|c|}{ Responses (n, \%) } \\
\hline & $\begin{array}{c}\text { Not at } \\
\text { all/A } \\
\text { little bit }\end{array}$ & $\begin{array}{l}\text { Mode- } \\
\text { rately }\end{array}$ & $\begin{array}{c}\text { Quite a } \\
\text { bit/Extremely }\end{array}$ \\
\hline $\begin{array}{l}\text { Did you like } \\
\text { the VW? }\end{array}$ & $0(0)$ & $\begin{array}{l}3 / 14 \\
(21)\end{array}$ & $11 / 14(79)$ \\
\hline $\begin{array}{l}\text { Did you } \\
\text { have } \\
\text { trouble } \\
\text { resting with } \\
\text { the VW? }\end{array}$ & $\begin{array}{c}13 / 14 \\
(93)\end{array}$ & $1 / 14(7)$ & $0(0)$ \\
\hline $\begin{array}{l}\text { Was the } \\
\text { VW } \\
\text { distracting } \\
\text { to you } \\
\text { during your } \\
\text { stay? }\end{array}$ & $\begin{array}{c}12 / 14 \\
(86)\end{array}$ & $1 / 14(7)$ & $1(7)$ \\
\hline & With a & $\mathbf{W}$ & ithout a VW \\
\hline $\begin{array}{l}\text { When given } \\
\text { the } \\
\text { opportunity } \\
\text { to have a } \\
\text { room with a } \\
\text { VW versus a } \\
\text { standard } \\
\text { room } \\
\text { without a } \\
\text { VW in the } \\
\text { ED, what } \\
\text { would you } \\
\text { prefer? }\end{array}$ & $11 / 14$ & & $3 / 14(21)$ \\
\hline
\end{tabular}

\section{Discussion}

Patients spend hours in the hospital without access to the outdoors and natural light. The lack of exposure to natural light results in increased risk of delirium, debilitating disorientation, and decreased satisfaction with the hospital experience. These effects are particularly prominent in the ED where continuous white light and noise prevent exposure to cues that may help orient patients who have extended ED stays. With increasing use of ED-based observation units where patients may spend up to two days in the ED for expedited management of acute and chronic medical problems, there is a need to provide improved orientation and exposure to the outdoors.

Our study demonstrates that virtual windows are an innovative technology that patients like, and may improve patient experience and provide sensory, orientation feedback. This is important because this intervention may address the universal problem of disorientation and its adverse events within the hospital. Unlike other interventions that may be targeted towards a specific disease, the use of a virtual window is innovative and distinctive as it uniquely integrates into the physical footprint of the ED and provides outdoor exposure to address commonalities in disorientation. We found that participants in the study were accepting of the virtual windows and willing to interact with them. Additionally, participants reported that they would prefer to be triaged to an ED room with a virtual window installed than a standard room without. This shows that more widespread deployment of virtual windows within the ED and other similar spaces may be an acceptable next step after this pilot.

We completed a limited pilot deployment to rapidly understand the use of virtual windows in the ED. In this context, we utilized a pre-recorded video stream. Future iterations of these systems should consider using live streams of outdoor settings. This can be accomplished by connecting the virtual window to a web camera, security camera or other public city cameras. Use of live cameras may provide distinct advantages as they may impart local context around weather conditions which could improve orientation. Conversely, the selection of calming scenes or sunny scenes may affect mood of ED patients. Additional innovations may include providing togglable scenes that the patient can select, or a physician may "prescribe" in the setting of delirium, disorientation, or decreased mood. Hospital environments must protect patient's privacy. Connected devices including live video cameras can have security vulnerabilities which could lead to inappropriate content being displayed on the virtual window or could enable 
access to the hospital network, including patient information. Virtual windows and their connected video cameras must therefore follow the highest information security standards and best practices. The use of a delayed live feed or looped content may impart some measure of control over video presented on a virtual window.

While our pilot study demonstrated the feasibility and acceptability of virtual windows among ED patients, the study is limited by the small number of participants. A future, larger study containing a matched control group may be able to demonstrate if exposure to a virtual window significantly improves patient experience, satisfaction, and orientation during ED hospitalization. Although it may be hypothesized that virtual windows have the potential to reduce delirium, agitation, and insomnia, this effect may be difficult to prove given the multi-factorial nature of these disease states. The use of various types of stimuli displayed on the virtual window may alter patient experiences and orientation. Finally, future studies should investigate the impact of virtual windows on patients with serious psychiatric disease. Often, these vulnerable patients may wait for days in a windowless environment while waiting for inpatient psychiatric hospitalization. These windows may provide the necessary stimuli to help reduce agitation and provide a connection to the outdoors.

\section{Funding}

Brigham Care Redesign Incubator Startup (BCRISP) Grant; PRC funded by NIH K23DA044874, R44DA051106, Hans and Mavis Psychosocial Foundation.

\section{References}

[1] J. Iwamoto et al., "Lower Incidence of In-Hospital Falls in Patients Hospitalized in Window Beds Than
Nonwindow Beds," J. Am. Med. Dir. Assoc., vol. 21, no. 4, pp. 476-480, Apr. 2020.

[2] M. Boubekri, I. N. Cheung, K. J. Reid, C.-H. Wang, and P. C. Zee, "Impact of Windows and Daylight Exposure on Overall Health and Sleep Quality of Office Workers: A Case-Control Pilot Study," J. Clin. Sleep Med. JCSM Off. Publ. Am. Acad. Sleep Med., vol. 10, no. 6, pp. 603-611, Jun. 2014.

[3] C. Blume, C. Garbazza, and M. Spitschan, "Effects of light on human circadian rhythms, sleep and mood," Somnologie, vol. 23, no. 3, pp. 147-156, 2019.

[4] M. Shepley, R. Gerbi, A. Watson, S. Imgrund, and R. Zadeh, "The Impact of Daylight and Views on ICU Patients and Staff," HERD, vol. 5, pp. 46-60, Nov. 2012.

[5] R. M. Buijs, C. Escobar, and D. F. Swaab, "The circadian system and the balance of the autonomic nervous system," Handb. Clin. Neurol., vol. 117, pp. 173-191, 2013.

[6] L. M. Wilson, "Intensive Care Delirium: The Effect of Outside Deprivation in a Windowless Unit," Arch. Intern. Med., vol. 130, no. 2, pp. 225-226, Aug. 1972.

[7] R. Smonig et al., "Impact of natural light exposure on delirium burden in adult patients receiving invasive mechanical ventilation in the ICU: a prospective study," Ann. Intensive Care, vol. 9, no. 1, p. 120, Oct. 2019.

[8] B. E. Karlin and R. A. Zeiss, "Best Practices: Environmental and Therapeutic Issues in Psychiatric Hospital Design: Toward Best Practices," Psychiatr. Serv., vol. 57, no. 10, pp. 1376-1378, Oct. 2006.

[9] M. Pettus, R. Cella, D. Urschel, and P. Dutta, "BMJ Winter 2016_Enhancing Cognitive PsychoPhysiology Virtual Windows," vol. 20, no. 1, pp. 39, Winter 2016.

[10] L. D. Rosen, K. Whaling, L. M. Carrier, N. A. Cheever, and J. Rokkum, "The Media and Technology Usage and Attitudes Scale: An empirical investigation," Comput. Hum. Behav., vol. 29, no. 6, pp. 2501-2511, Nov. 2013.

[11] S. Cohen, T. Kamarck, and R. Mermelstein, "A Global Measure of Perceived Stress," J. Health Soc. Behav., vol. 24, no. 4, pp. 385-396, 1983. 